\title{
Dynamics and heating of the magnetic network on the Sun Efficiency of mode transformation
}

\author{
S. S. Hasan ${ }^{1}$ and P. Ulmschneider ${ }^{2}$ \\ ${ }^{1}$ Indian Institut of Astrophysics, Bangalore-560034, India \\ e-mail: hasan@iiap.res.in \\ 2 Institut für Theoretische Astrophysik, Tiergartenstr. 15, 69121 Heidelberg, Germany \\ e-mail: ulm@ita.uni-heidelberg.de
}

Received 5 February 2004 / Accepted 20 April 2004

\begin{abstract}
We aim to identify the physical processes which occur in the magnetic network of the chromosphere and which contribute to its dynamics and heating. Specifically, we study the propagation of transverse (kink) MHD waves which are impulsively excited in flux tubes through footpoint motions. When these waves travel upwards, they get partially converted to longitudinal waves through nonlinear effects (mode coupling). By solving the nonlinear, time-dependent MHD equations we find that significant longitudinal wave generation occurs in the photosphere typically for Mach numbers as low as 0.2 and that the onset of shock formation occurs at heights of about $600 \mathrm{~km}$ above the photospheric base. We also investigate the compressional heating due to longitudinal waves and the efficiency of mode coupling for various values of the plasma $\beta$, that parameterises the magnetic field strength in the network. We find that this efficiency is maximum for field strengths corresponding to $\beta \approx 0.2$, when the kink and tube wave speeds are almost identical. This can have interesting observational implications. Furthermore, we find that even when the two speeds are different, once shock formation occurs, the longitudinal and transverse shocks exhibit strong mode coupling.
\end{abstract}

Key words. Sun: magnetic fields - Sun: oscillations - Sun: chromosphere

\section{Introduction}

The solar chromosphere plays an important role as the lower boundary of the heliosphere, as the source of the solar XUV radiation which affects the ionisation state of the upper terrestrial atmosphere as well as for the origin of the solar wind and the generation of coronal mass ejections. It is thus of great interest to understand the chromosphere and, in particular, the state of the gas in its upper layers. In the quiet chromosphere one needs to distinguish between the magnetic network and internetwork regions in the cell interior. The former occurs on the boundary of several supergranulation cells which are the sites of the ubiquitous Network Bright Points (NBPs) in which strong magnetic fields are organized in magnetic flux tubes. On the other hand, magnetic fields are weak and dynamically unimportant in the internetwork.

The focus of this investigation is to examine wave propagation in magnetic elements of the network. Several observations have revealed that network oscillations have periods that are much longer than those in internetwork regions (Lites et al. 1993; Judge et al. 1997; Curdt \& Heinzel 1998; Cauzzi et al. 2000). A careful analysis of NBPs has revealed that these oscillations possess significant power in the $1-4 \mathrm{mHz}$
(Banerjee et al. 2001; McAteer et al. 2002, 2003; Bloomfield et al. 2004), along with evidence which suggests the existence of multiple peaks.

A theoretical understanding of the waves observed in the network is far from complete. An idealized scenario is to treat the network as a conglomeration of thin vertical magnetic flux tubes fanning out with height. It is well known that such flux tubes support a variety of wave modes: the sausage or longitudinal mode, the kink or transverse mode, and the torsional Alfvén mode (Spruit 1982; Roberts \& Ulmschneider 1997). This approach was adopted by Musielak et al. $(1989,1995)$, Huang et al. (1995) and Ulmschneider \& Musielak (1998), Musielak \& Ulmschneider (2001, 2002, 2003a,b), Noble et al. (2003) to examine MHD wave generation in flux tubes through an interaction with turbulent motions in the convection zone. In these studies, the turbulence was characterised using a modified Kolmogorov spectrum. An alternative model, based on $G$-band observations of NBPs motions (Muller 1983, 1985), investigated the excitation of MHD kink waves in flux tubes through impulsive motions of their footpoints (Choudhuri et al. 1993).

The latter hypothesis was investigated in greater detail by Hasan \& Kalkofen (1999) who examined the generation of transverse and longitudinal waves in a flux tube through 
buffeting by granular motions in the magnetic network. It was found that the generic response of the flux tube to a single granular impact is the same for both transverse and longitudinal waves: the buffeting action excites a pulse that propagates along the flux tube with the kink or longitudinal tube speed. For strong magnetic fields, most of the energy goes into transverse waves, and only a much smaller fraction into longitudinal waves. After the passage of the pulse, the atmosphere gradually relaxes to a state in which it oscillates at the cutoff period of the mode. The period observed in the magnetic network was interpreted as the cutoff period of transverse waves, which leads naturally to an oscillation at this period (typically in the 7-min range) as proposed by Kalkofen (1997).

As a continuation of the above investigation, Hasan et al. (2000) modeled the excitation of waves in the network due to the observed motions of $G$-band bright points, which were taken as a proxy for footpoint motions of flux tubes. For a typical magnetic element in the network they predicted that the injection of energy into the chromosphere takes place in brief and intermittent bursts, lasting typically $30 \mathrm{~s}$, separated by long periods with low energy flux; this implies a high intermittency in chromospheric emission, which may be incompatible with observations. They concluded that there must be other highfrequency motions (periods 5-50 s) which cannot be detected as proper motions of $G$-band bright points. Adding such highfrequency motions to their simulations they obtained much better agreement with the persistent emission observed from the magnetic network. They speculated that the high-frequency motions could be due to turbulence in intergranular lanes. This idea, however, requires further investigation.

The above studies were based on a linear approximation in which the longitudinal and transverse waves are decoupled. However, it is well known that the velocity amplitude $v(z)$ for the two modes increases with height $z$ (for an isothermal atmosphere $v \propto \exp [z / 4 H]$, where $H$ is the pressure scale height), so that the motions are likely to become supersonic higher up in the atmosphere. At such heights, nonlinear effects become important, leading to a coupling between the transverse and longitudinal modes. Some progress on this question has been made in one dimension (1-D) using the nonlinear equations for a thin flux tube (Ulmschneider et al. 1991; Huang et al. 1995), Zhugzhda et al. (1995) and more recently by Hasan et al. (2003, hereafter Paper I).

In Paper I, the nature of mode coupling between the transverse and longitudinal modes in the magnetic network was investigated in some detail. They modelled the excitation of kink oscillations through impulsive footpoint motions of flux tubes and found that when the transverse velocities are significantly less than the kink wave speed (the linear regime), there is essentially no excitation of longitudinal waves. However, at heights where the Mach number of the kink oscillations is around 0.3, longitudinal modes begin to be excited. Longitudinal wave generation becomes most efficient at Mach numbers around unity, leading to the modes having comparable amplitudes. A comparison of the results with the exact linear solution for transverse waves enabled them to locate the regions in the atmosphere where nonlinear effects are important.
The main aim of the present study is to extend the calculations of Paper I to examine the efficiency of mode conversion for different values of $\beta$, which essentially is a measure of the magnetic field strength in the magnetic network. The motivation for such a study stems from trying to understand whether mode conversion would be efficient over a wide range of $\beta$ values or whether it holds only for a narrow range of $\beta$. In our scenario, the heating of the network elements is assumed to occur through the dissipation of longitudinal waves, which are generated from transverse waves through mode conversion. Therefore, our investigation could be useful in placing constraints on the field strengths in the network where chromospheric heating occurs through the above process.

The organization of this paper is as follows: in Sect. 2 we discuss the excitation of MHD waves by footpoint motions in a vertical "thin" flux tube. along with the initial equilibrium model and the method of solution. The results of our calculation are presented in Sect. 3 followed by a discussion and summary in Sect. 4, where we also point out some observational implications of our investigation.

\section{Wave excitation due to footpoint motion}

In this section we study wave propagation in a tube over a height range where the thin-flux tube approximation (TFA) can be applied. The validity of this approximation to flux tubes in the solar atmosphere is discussed in some detail in Paper I. By comparing the structure of a thin flux tube with that calculated using a 2-D model, it was shown that the TFA can be applied up to heights of around $1000 \mathrm{~km}$ above the photospheric base. At larger heights, the TFA deteriorates owing to the expansion of the flux tube near the "canopy".

\subsection{Initial flux tube model}

Similar to Paper I, let us consider a flux tube extending vertically through the photosphere and chromosphere of the Sun. We assume that the flux tube is "thin" and initially in hydrostatic equilibrium and isothermal, with the same temperature as the external medium, which we take to be $6000 \mathrm{~K}$. We consider a tube with a radius of $50 \mathrm{~km}$ at $z=0$. The radius increases with $z$ as $\exp (z / 4 H)$ in an isothermal atmosphere for a thin flux tube.

\subsection{Method of solution}

The basic equations for adiabatic longitudinal-transverse MHD waves in a thin flux tube consist of a set of coupled differential equations (see Ulmschneider et al. 1991, for details) which are solved numerically using the method of characteristics. Our method is based on a modified version of the code developed by Ulmschneider et al. (1991) that treats shocks (following Zhugzhda et al. 1995) as well as the back reaction of the external fluid on the tube (Osin et al. 1999). The code is capable of resolving shocks in the chromosphere. However, we have made an important modification to this code by incorporating the transmission of strong shocks through the upper boundary. The computational domain in the vertical direction 


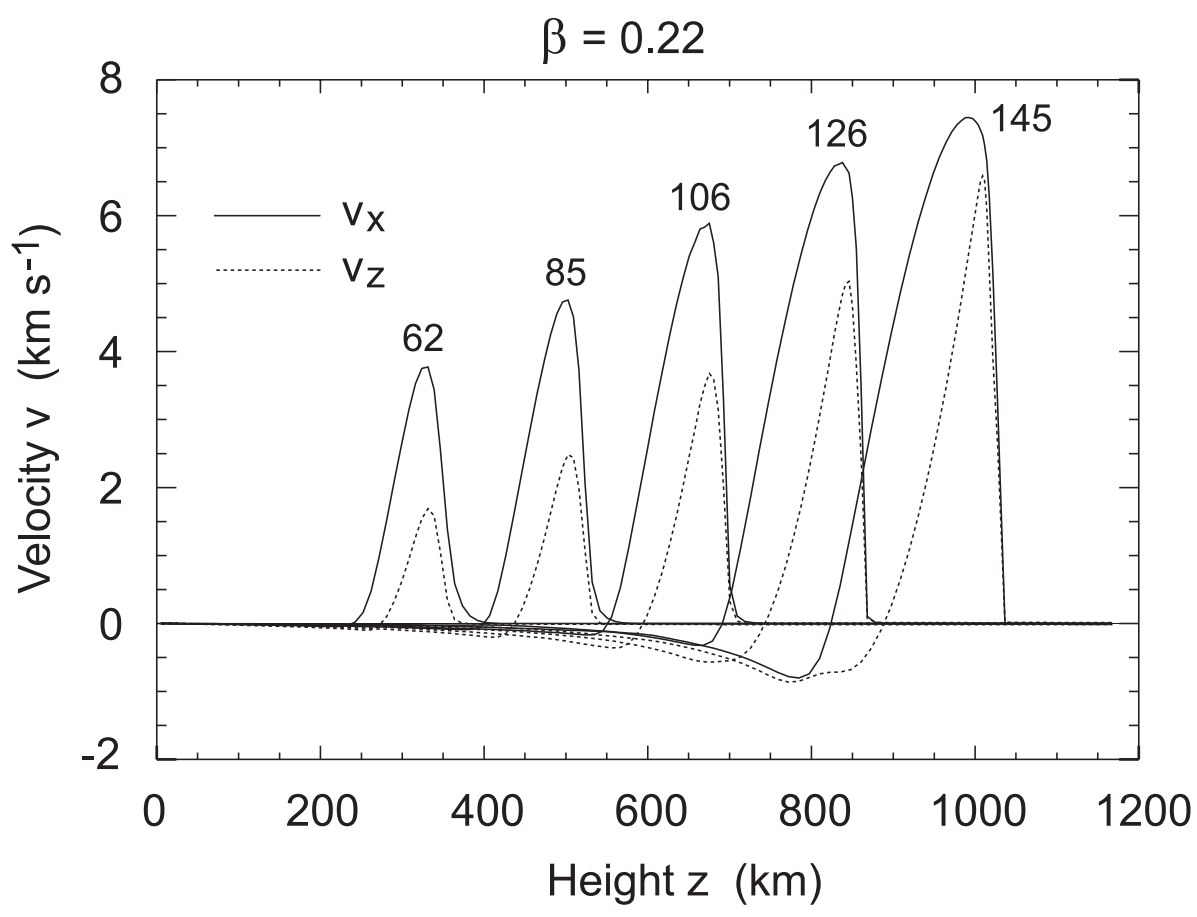

Fig. 1. Nonlinear coupling of transverse and longitudinal waves in a flux tube: transverse velocity $v_{x}$ (solid curves) and longitudinal velocity $v_{z}$ (dashed curves) as functions of height $z$ at various times, for $\beta=0.22, t_{0}=20 \mathrm{~s}, \tau=5 \mathrm{~s}, v_{0}=2.5 \mathrm{~km} \mathrm{~s}^{-1}$. The numbers adjacent to the curves denote the time in seconds.

has an equidistant grid of size about $9 \mathrm{~km}$. The Courant condition is used to select the time step to advance the equations in time.

\subsection{Boundary conditions}

At the lower boundary, taken at $z=0$, we assume that the flux tube has a transverse footpoint motion consisting of a single impulse of the form:

$v_{x}(0, t)=v_{0} \mathrm{e}^{-\left[\left(t-t_{0}\right) / \tau\right]^{2}}$

where $v_{0}$ is the specified velocity amplitude, $t_{0}$ denotes the time when the motions have maximum amplitude and $\tau$ is the time constant of the impulse. The longitudinal component of the velocity at the base is assumed to be zero. In the present calculations we take $v_{0}=2.5 \mathrm{~km} \mathrm{~s}^{-1}, t_{0}=20 \mathrm{~s}$ and $\tau=5 \mathrm{~s}$.

At the upper boundary of the computational domain (taken at $z=1200 \mathrm{~km}$ ) we use transmitting boundary conditions, following Ulmschneider et al. (1977), and assume that the velocity amplitude remains constant along the outward-propagating characteristics. We use the characteristic equations to selfconsistently determine physical quantities at the boundary.

\section{Results}

The initial equilibrium model is perturbed with a transverse motion at $z=0$ in the form of an impulse with a velocity given by Eq. (1). This impulse generates a transverse wave that propagates upwards with the kink wave tube speed. The resulting motion in the tube as a function of height and time follows from the time-dependent MHD equations for a thin flux tube.
Figure 1 shows the variation of the transverse $v_{x}$ (solid lines) and longitudinal $v_{z}$ (dashed lines) components of the velocity as a function of height $z$ at various epochs of time $t$ for a magnetic field strength of $1425 \mathrm{G}$ at $z=0$, that corresponds to a plasma $\beta=0.22$ (which by assumption remains constant with height for a thin flux tube). In the present case, the kink and longitudinal tube speeds are approximately the same with a value of about $7.3 \mathrm{~km} \mathrm{~s}^{-1}$. The numbers next to the curves denote the time $t$ (in s). We find that low in the atmosphere, where the transverse velocity amplitude is small (compared to the kink wave speed $c_{\mathrm{K}}$ ), the longitudinal component of the velocity is negligible. As the initial pulse propagates upwards, the transverse velocity amplitude increases. Due to nonlinear effects, longitudinal motions are generated. We find that even at fairly low heights $(z \approx 300 \mathrm{~km})$, the latter are significant for a Mach number $M=v_{x} / c_{\mathrm{K}}$ as small as 0.2 . The longitudinal pulse propagates at the tube speed $c_{\mathrm{T}}$, which in the present case is almost identical with $c_{\mathrm{K}}$. The efficiency of the nonlinear coupling increases with the amplitude of the transverse motions. When $v_{x} \approx c_{\mathrm{K}}$, the amplitudes in the transverse and longitudinal components become comparable. The longitudinal motions, being compressive, steepen with height and eventually form shocks. A shock is first formed at a height of about $600 \mathrm{~km}$. This is a kink-longitudinal shock as discussed in detail by Zhugzhda et al. (1995), which travels upward with the common propagation kink/tube speed. We expect the compression associated with the longitudinal shock to produce heating as we shall see in the next figure.

Figures $2 a-d$ depict the height variation of the horizontal and vertical components of the velocity (solid and dotted lines respectively) and the temperature $T$ (dashed line) at a 

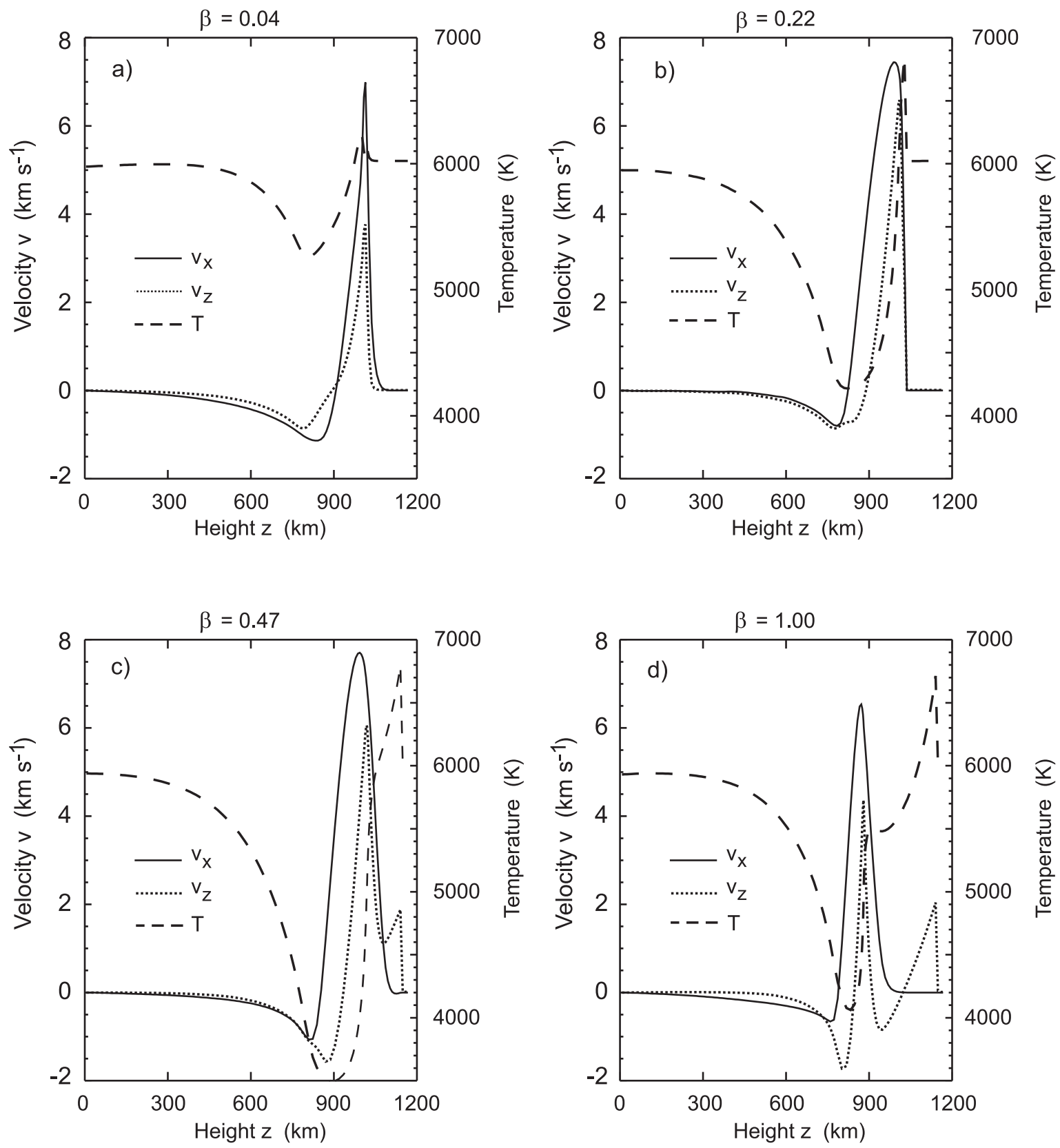

Fig. 2. Snapshots of the transverse velocity $v_{x}$ (solid curves) and longitudinal velocity $v_{z}$ (dotted curves) and the temperature $T$ (dashed curves) as functions of height $z$ for (a) $\beta=0.04$, (b) $\beta=0.22$, (a) $\beta=0.47$, and (d) $\beta=1.00$.

certain instant of time for various values of $\beta$. In Fig. 2a, we find that due to mode coupling, the transverse and longitudinal shocks travel approximately together, despite the fact that the phase speeds for the two modes are different $\left(c_{\mathrm{K}}=7.9 \mathrm{~km} \mathrm{~s}^{-1}\right.$ and $c_{\mathrm{T}}=8.5 \mathrm{~km} \mathrm{~s}^{-1}$ ). In Fig. 2b, we find that the location of the shocks occurs at identical heights, which is not surprising since in this case $c_{\mathrm{K}}=7.9 \approx c_{\mathrm{T}}=7.3 \mathrm{~km} \mathrm{~s}^{-1}$. As we increase $\beta$ further, so that $c_{\mathrm{K}}<c_{\mathrm{T}}$, an interesting phenomenon occurs. The longitudinal shock splits into two shocks, one occurring at about the same location as the kink shock, but another one of smaller magnitude occurring at a greater height. The latter travels with the speed $c_{\mathrm{T}}$.

We now consider the effect of the waves on the temperature structure in the flux tube. Through nonlinear interactions, longitudinal waves are generated from purely transverse wave excitation. Being compressive, longitudinal waves heat the atmosphere. The dashed curves in Figs. $2 \mathrm{a}-\mathrm{d}$ show the temperature enhancement due to the compressive longitudinal waves. In Figs. $2 \mathrm{a}$ and $2 \mathrm{~b}$ corresponding to strong magnetic fields for which $c_{\mathrm{K}}>c_{\mathrm{T}}$, the amplitude of the temperature pulse increases with the longitudinal velocity amplitude. However, the situation is more complicated for weaker fields $\left(c_{\mathrm{K}}<c_{\mathrm{T}}\right)$. From Figs. $2 \mathrm{c}$ and $2 \mathrm{~d}$, we find that the shock heating does not occur at the common transverse-longitudinal wave front but rather at a larger height associated with a longitudinal shock.

Finally, we examine the efficiency of the mode coupling as a function of $\beta$, which parameterises the magnetic field strength in the flux tube. The mode coupling efficiency at a particular 


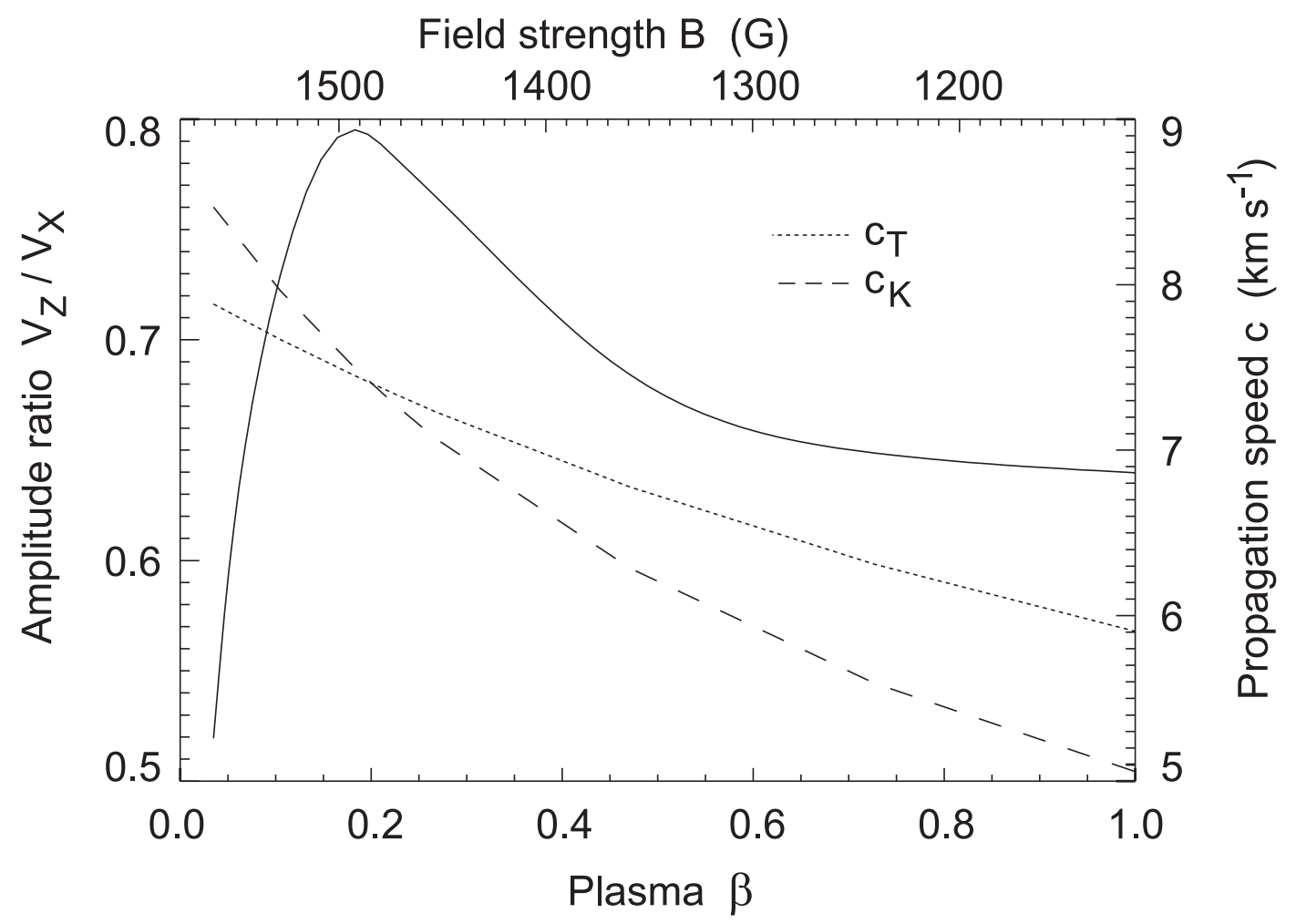

Fig. 3. Efficiency of nonlinear coupling between transverse and longitudinal waves in a flux tube: $v_{z} / v_{x}$ (solid curves) at $z=900 \mathrm{~km}, c_{\mathrm{K}}(\mathrm{dashed}$ curve) and $c_{\mathrm{T}}$ (dotted curve) as functions of $\beta$. The field strength $B$ at $z=0$ is given by the top axis.

height can be measured in terms of the ratio $v_{z} / v_{x}$, since the longitudinal motions are essentially generated from the transverse motions through nonlinear interactions. Figure 3 shows the variation of $v_{z} / v_{x}$ with $\beta$ at $z=900 \mathrm{~km}$. The top scale shows the corresponding values of the magnetic field strength at $z=0$. The dashed and dotted lines denote the kink and tube speeds respectively (with respect to the scale on the right). For low values of $\beta$ (i.e. for which $c_{\mathrm{K}}>c_{\mathrm{T}}$ ) the efficiency increases rapidly with $\beta$ reaching a maximum at $\beta \approx 0.2$ i.e., when the kink and tube speeds are equal. When $c_{\mathrm{T}}>c_{\mathrm{K}}$, the efficiency drops with increasing $\beta$. At large values of $\beta, v_{z} / v_{x}$ appears to level off to a limiting value.

\section{Discussion and summary}

The results presented in the previous section have highlighted several interesting features connected with wave propagation in magnetic flux tubes. Similar to Paper I, we found that through the forcing action of purely transverse motions at the base of a flux tube, longitudinal motions are generated higher up in the tube through mode coupling. The new refinements in our present code, allow us to accurately follow shocks as well as to examine the coupling of the modes to large heights in the atmosphere.

\subsection{Linear mode coupling at similar propagation speeds}

The focus of this work has been on examining the effect of $\beta$ on the mode coupling between the transverse and longitudinal waves in the solar atmosphere. It is, of course well known, that a magnetized atmosphere exhibits coupled slow and fast modes which for a low $\beta$ plasma are akin to acoustic and magnetic modes respectively. Interesting effects occur when $\beta$ varies with height in the atmosphere. Bogdan et al. (2003) point out that the $\beta=1$ layer, which they identify with the magnetic canopy, is crucial in understanding the nature of MHD wave propagation in the solar chromosphere. In this layer, the fast and slow MHD waves experience strong coupling which leads to "mode mixing" (e.g., Campos 1987; Alicki et al. 1994). It should be noted that this coupling is essentially a linear effect which is due to the change in the relative strengths of the sound and Alfvén speeds in the atmosphere. We should like to clarify that in the framework of the thin flux tube approximation, however, there is no linear coupling between the transverse and longitudinal modes. The coupling that we discuss in this paper arises solely due to nonlinear effects. The physical origin of this phenomenon has been discussed by Ulmschneider et al. (1991) (see also Ulmschneider 2003).

\subsection{Nonlinear mode coupling of waves and shocks}

Let us now consider the non-linear mode coupling between the transverse and longitudinal waves. In this context we would like to distinguish between two effects: (a) the generation of longitudinal waves through purely transverse displacements at the base of the flux tube and; (b) the coupling between longitudinal and kink shocks. The first effect has already been discussed in Paper I as well as by Ulmschneider et al. (1991) or Ulmschneider (2003) and no further elaboration is necessary, other than the fact that its efficiency is highest when the tube and kink waves speeds become identical. 
On the other hand, even when the two speeds are different, so that the nonlinear interaction at lower wave amplitude (see Fig. 2a) is less efficient, we find that as soon as the shock forms, the transverse shock and the longitudinal shock stay at the same height and propagate with the same common speed, different from both the longitudinal and transverse wave speeds. This new property of shock waves suggests a particularly large and unusual amount of mode coupling. It has already been found in earlier calculations (Zhugzhda et al. 1995; Hollweg et al. 1982). The fact that this feature seems to be a fundamental property of shock waves and is not an artifact of our wave computation can be seen by noting that it also occurs in other time-dependent MHD wave computations using different wave modes and other mathematical methods. We should point out that our computations are based on the method of characteristics where shocks are treated as discontinuities at which the two sides are connected with the help of the Hugoniot relations whereas the treatment of Hollweg et al. use a finite difference method where the treatment of shocks is not different from that of other interior points.

Figures 3 and 4 by Hollweg et al. (1982) show the propagation of torsional wave pulses in a solar magnetic flux tube. These authors examined the time development of wave pulses of $90 \mathrm{~s}$ duration with different initial torsional velocity amplitudes. It is seen that these pulses propagate towards greater height and eventually develop strong torsional shocks (called switch-on shocks). Mode coupling also generates longitudinal waves. A comparison of the shock position of the torsional and longitudinal shocks at the various times shows that the shocks are always at the same height and propagate at the same common speed, exactly like the behaviour which we found in Fig. 2 for our coupled longitudinal-transverse wave system.

\subsection{Mode coupling and heating}

Let us now turn to the question of wave heating associated with the motions in the flux tube. As can be seen from Figs. $2 \mathrm{a}-\mathrm{d}$, heating takes place in the tube due to the occurrence of shocks. For very small values of $\beta$, when the kink wave speed $c_{\mathrm{K}}>c_{\mathrm{T}}$, we find that shock heating is minimal. The largest amount of shock heating at the common kink-longitudinal shock occurs when the transverse and longitudinal wave speeds are approximately identical, and where $v_{z} \approx v_{x}$. For weaker fields $\left(c_{\mathrm{K}}<c_{\mathrm{T}}\right)$, the heating at the common shock front again becomes negligible. However, in this case, the presence of the additional purely longitudinal shock produces heating ahead of the kink-longitudinal shock. This is another new feature to emerge from the present calculations. We should clarify that although we use an adiabatic energy equation that assumes entropy conservation in the flux tube, we use the RankineHugoniot relations at the shock front to connect the pre and post shock states. It is the entropy jump at the shock front which is responsible for the heating discussed above.

It would be premature for us to estimate, on the basis of the calculations in this paper, the wave energy flux entering the corona or even the upper chromosphere. This is mainly on account of two assumptions that have been made by us. The first one is our choice of an isothermal atmosphere which implies that the pressure scale height and the sound speed are constant with height $z$. For the height range considered by us, the sound speed variation is less than $10 \%$ and is unlikely to affect the results in a fundamental way.

The second assumption which we have made that is more serious is the thin flux tube approximation, whose validity was carefully examined in Paper I. By comparing the exact magnetostatic solution for an axisymmetric flux tube, with that obtained using the thin flux tube approximation, it was shown that the latter is reasonable up to heights of about $1 \mathrm{Mm}$ above the photosphere for tubes with a filling factor less than $1 \%$. on the solar surface. At larger heights even a sufficiently thin flux tube expands rapidly and comes in contact with neighbouring tubes, making the problem intractable within the present formalism. In such a situation, where further spreading of the tube is inhibited, $\beta$ is no longer constant but typically increases with height and the shape of the tube in the upper atmosphere resembles a "wine glass". However, in our investigation we have used an upper boundary at a location where the exponentially diverging tube behaviour approximates the true solution reasonably well.

\subsection{Mode coupling in the wine glass geometry}

A major difficulty of using a modified thin flux tube with a "wine glass" in the upper chromosphere stems from the difficulty of including the effects of waves from different flux tubes interfering with one another at such locations. Clearly, one needs to resort to multidimensional calculations to treat the problem accurately. Recently, some progress has been made in this direction (Rosenthal et al. 2002; Bogdan et al. 2003) who have carried out numerical simulations of wave propagation in 2-D, assuming that the displacements lie in a plane perpendicular to the invariant direction, for magnetic geometries representative of the network and inter-network regions on the Sun. The velocity amplitudes in the above simulations are sufficiently small so that nonlinear effects are relatively unimportant compared to those associated with the field geometry. Furthermore, these waves are different from the kink and sausage modes considered by us, for which three-dimensional simulations would be required, that have not yet been carried out. Nevertheless, the above work represents an important step which should be followed up by more refined calculations to allow a realistic comparison with observations.

\subsection{Observational implications}

It is interesting to speculate on the observational implications of our calculations. Observationally, it is hard to unambiguously test whether there is evidence of mode coupling in the network based on the scenario presented by us. McAteer et al. (2003) and Bloomfield et al. (2004) examined several NBPs and inferred the possibility of mode coupling between transverse and longitudinal modes in about a third of them. This inference was based on cross-correlating power in different lines to look for signatures of transverse and longitudinal modes at different heights in the atmosphere. In the lower atmosphere, the modes are dominantly transverse with a frequency in the range $1.2-1.6 \mathrm{mHz}$ that can be identified with 
the kink cutoff frequency. Through mode coupling, longitudinal waves are generated at double the frequency (e.g. Musielak \& Ulmschneider 2003b). On the basis of the present theoretical study, it is difficult to predict the dominant frequencies for the transverse and longitudinal waves in NBPs other than the presence of their respective cutoff frequencies (see Paper I) if the footpoint excitation occurs in the form of a single pulse. However, for a more realistic excitation mechanism, which on observationally grounds is likely to be stochastic in nature, further work is required before any predictions on mode frequencies can be made. We hope to examine this problem in greater detail in future work.

The present calculations would suggest that an investigation of a large sample of NBPs would tend to show weak mode coupling for very large field strengths (at the photospheric base) greater than about $1500 \mathrm{G}$. For weaker field strengths, the mode coupling efficiency would be correlated with the field strength at $z=0$. This may be consistent with why McAteer et al. (2003) and Bloomfield et al. (2004) did not find evidence of mode coupling in all the NBPs they examined. We expect that future observations will shed more light on this question.

In summary, the purpose of the present investigation was to examine the effect of the magnetic field strength (parameterised by $\beta$ ) on the dynamics, heating and efficiency of mode coupling in flux tubes that are typical of the magnetic network on the Sun. We modelled the excitation of kink oscillations through impulsive footpoint motions. Through nonlinear mode coupling, longitudinal motions are generated. For typical parameters in the network, we found that significant longitudinal wave generation occurs at heights as low as $300 \mathrm{~km}$ in the atmosphere and that shock formation occurs at about $z=600 \mathrm{~km}$, which can lead to heating of the chromosphere. The efficiency of mode coupling is maximum when the kink and tube waves speeds are almost identical, which occurs for $\beta \approx 0.2$. We also pointed out that the present calculations can have interesting observational implications. In future work we hope to overcome the limitations of the thin flux tube approximation to examine more thoroughly chromospheric heating and also the transmission of wave energy into the corona.

Acknowledgements. We have benefited from the valuable comments and suggestions of the referee Z. Musielak. S.S.H. gratefully acknowledges support from the Deutsche Forschungs Gemeinschaft to visit the Institut für Theoretische Astrophysik, University of Heidelberg for carrying out the work reported in this paper. S.S.H. is thankful to K. Sivaraman for helpful discussions.

\section{References}

Alicki, R., Musielak, E. Z., Sikorski, J., \& Makowiec, D. 1994, ApJ, 427,919
Banerjee, D., O'Shea, E., Doyle, J. G., \& Goosens, M. 2001, A\&A, 357, 1093

Bloomfield, D. S., McAteer, R. T. J., Mathioudakis, M., Williams, D. R., \& Keenan, F. P. 2004, ApJ, 604, 936

Bogdan, T. J., Carlsson, M., \& Hansteen, V., et al. 2003, ApJ, 599, 626

Campos, L. M. B. C. 1987, Rev. Mod. Phys., 59, 363

Cauzzi, G., Falchi, A., \& Falciani, R. 2000, A\&A, 357, 1093

Choudhuri, A. R., Auffret, H., \& Priest, E. R. 1993, Sol. Phys., 143, 49

Curdt, W., \& Heinzel, P. 1998, ApJ, 503, L95

Hasan, S. S., \& Kalkofen, W. 1999, ApJ, 519, 899

Hasan, S. S., Kalkofen, W., \& van Ballegooijen, A. A. 2000, ApJ, 535, L67

Hasan, S. S., Kalkofen, W., van Ballegooijen, A. A., \& Ulmschneider, P. 2003, ApJ, 585, 1138

Heinzel, P., \& Curdt, W. 1999, in Third Advances in Solar Physics Euroconference: Magnetic Fields and Oscillations, ed. B. Schmieder, A. Hofmann, \& J. Staude, ASP Conf. Ser., 184, 201

Hollweg, J. V., Jackson, S., \& Galloway, D. 1982, Sol. Phys., 75, 35

Huang, P., Musielak, Z. E., \& Ulmschneider, P. 1995, A\&A, 297, 579

Judge, P., Carlsson, M., \& Wilhelm, K. 1997, ApJ, 490, L195

Kalkofen, W. 1997, ApJ, 486, L148

Lites, B. W., Rutten, R. J., \& Kalkofen, W. 1993, ApJ, 414, 345

McAteer, R. T. J., Gallagher, P. T., Williams, D. R., et al. 2002, ApJ, $567, \mathrm{~L} 168$

Muller, R. 1983, Sol. Phys., 85, 113

Muller, R. 1985, Sol. Phys., 100, 237

Musielak, Z. E., \& Ulmschneider, P. 2001, A\&A, 370, 541

Musielak, Z. E., \& Ulmschneider, P. 2002, A\&A, 386, 606

Musielak, Z. E., \& Ulmschneider, P. 2003a, A\&A, 400, 1057

Musielak, Z. E., \& Ulmschneider, P. 2003b, A\&A, 406, 725

Musielak, Z. E., Rosner, R., \& Ulmschneider, P. 1989, ApJ, 337, 470

Musielak, Z. E., Rosner, R., Gail, H. P., \& Ulmschneider, P. 1995, ApJ, 448,865

Noble, M. W., Musielak, Z. E., \& Ulmschneider, P. 2003, A\&A, 409, 1085

Osin, A., Volin, S., \& Ulmschneider, P. 1999, A\&A, 351, 359

Roberts, B., \& Ulmschneider, P. 1997, in Solar and Heliospheric Plasma Physics, ed. G. M. Simett, C. E. Alissandrakis, \& L. Vlahos, Lect. Notes Phys., 489 (Berlin: Springer Verlag, Heidelberg), 75

Rosenthal, C. S., Bogdan, T. J., Carlsson, M., et al. 2002, ApJ, 564, 508

Spruit, H. C. 1982, Sol. Phys., 75, 3

Ulmschneider, P. 2003, in Lectures on Solar Physics, ed. H. M. Antia, A. Bhatnagar, \& P. Ulmschneider, Lecture Notes in Physics, 619 (Berlin: Springer Verlag, Heidelberg), 232

Ulmschneider, P., \& Musielak, Z. E. 1998, A\&A, 338, 311

Ulmschneider, P., Nowak, T., Bohn, U., \& Kalkofen, W. 1977, A\&A, 54,61

Ulmschneider, P., Zähringer, K., \& Musielak, Z. E. 1991, A\&A, 241, 625

Zhugzhda, Y. D., Bromm, V., \& Ulmschneider, P. 1995, A\&A, 300, 302 